\title{
Ultrasound-targeted microbubble destruction-mediated Ang1 gene transfection improves left ventricular structural and sympathetic nerve remodeling in canines with myocardial infarction
}

\author{
Sheng Cao", Qing Deng", Yijia Wang, Yanxiang Zhou, Qing Zhou \\ Department of Ultrasound Imaging, Renmin Hospital of Wuhan University, Wuhan, China \\ Contributions: (I) Conception and design: S Cao, Q Deng, Q Zhou; (II) Administrative support: S Cao, Q Deng, Y Wang, Q Zhou; (III) Provision of \\ study materials or patients: S Cao, Q Deng, Y Zhou; (IV) Collection and assembly of data: Y Wang, Y Zhou; (V) Data analysis and interpretation: S \\ Cao, Q Deng, Q Zhou; (VI) Manuscript writing: All authors; (VII) Final approval of manuscript: All authors. \\ \#These authors contributed equally to this work. \\ Correspondence to: Qing Zhou. Department of Ultrasound Imaging, Renmin Hospital of Wuhan University, 238\# Jiefang Road, Wuchang District, \\ Wuhan 430060, China. Email: qingzhou.wh.edu@hotmail.com.
}

Background: The present study aimed to determine whether ultrasound-targeted microbubble destruction (UTMD)-mediated angiopoietin 1 (Ang1) gene transfection can improve angiogenesis and potentially reverse left ventricular (LV) structural and sympathetic nerve remodeling in canines with myocardial infarction (MI). Methods: Thirty dogs were randomly divided into groups ( $\mathrm{n}=10$ /group) as follows: the MI group (MI dogs without UTMD treatment), the UTMD group (MI dogs with UTMD-mediated negative control plasmid treatment), and the UTMD-Ang1 group (MI dogs with UTMD-mediated Ang1 plasmid treatment). LV dimensions, systolic function, and synchrony were used to reflect the structural remodeling. The density of tyrosine hydroxylase (TH)- and growth-associated protein 43 (GAP43)-positive nerve fibers were calculated to assess the sympathetic nerve remodeling.

Results: One month after treatment, the UTMD-Ang1 group showed lower LV end-diastolic dimension (LVEDD: $31.2 \pm 2.3 \mathrm{~mm}$ ) and higher LV ejection fraction (LVEF: $44.6 \% \pm 4.3 \%$ ) than the MI group (LVEDD: $34.5 \pm 2.2 \mathrm{~mm}, \mathrm{t}=2.282, \mathrm{P}=0.014$; LVEF: $37.3 \% \pm 3.1 \%, \mathrm{t}=3.718, \mathrm{P}=0.003$ ) and the UTMD group (LVEDD: $34.1 \pm 2.8 \mathrm{~mm}, \mathrm{t}=2.264, \mathrm{P}=0.040$; LVEF: $39.3 \% \pm 4.5 \%, \mathrm{t}=2.408, \mathrm{P}=0.030) . \mathrm{LV}$ synchrony was higher in the UTMD-Ang1 group compared with the MI group by 2-dimensional speckle-tracking echocardiography. Angiogenic density was higher in the UTMD group than the MI group but was highest in the UTMD-Ang1 group according to immunohistochemistry of CD31 and $\alpha$-smooth muscle actin staining. The density of THand GAP43-positive nerve fibers were decreased in the UTMD-Ang1 group (TH: $1,928.2 \pm 376.6 \mu \mathrm{m}^{2} / \mathrm{mm}^{2}$; GAP43: 2,090.8 $\pm 329.2 \mu \mathrm{m}^{2} / \mathrm{mm}^{2}$ ) compared with the MI group (TH: $2916.5 \pm 558.4 \mu \mathrm{m}^{2} / \mathrm{mm}^{2}$, $\mathrm{t}=4.069, \mathrm{P}=0.001$; GAP43: 3,275.4 $\left.\pm 548.6 \mu \mathrm{m}^{2} / \mathrm{mm}^{2}, \mathrm{t}=5.153, \mathrm{P}=0.000\right)$ and the UTMD group (TH: 2,552.7 $\pm 408.1 \mu \mathrm{m}^{2} / \mathrm{mm}^{2}, \mathrm{t}=3.181, \mathrm{P}=0.007$; GAP43: 2,630.5 $\left.\pm 419.3 \mu \mathrm{m}^{2} / \mathrm{mm}^{2}, \mathrm{t}=2.863, \mathrm{P}=0.013\right)$. The relative Ang1 and sarcoplasmic reticulum $\mathrm{Ca}^{2+}$-ATPase 2a protein levels were significantly higher in the UTMD-Ang1 group than the UTMD and MI groups by Western blot, while the phospholamban levels exhibited the opposite trend. Plasma norepinephrine and N-terminal pro-B-type-natriuretic peptide were significantly reduced in the UTMD-Ang1 group from day 1 to 1 month after MI.

Conclusions: UTMD-mediated Ang1 transfection can promote angiogenesis, reverse LV structural and sympathetic nerve remodeling, and improve LV synchrony after MI.

Keywords: Ultrasound-targeted microbubble destruction (UTMD); gene transfection; myocardial infarction; left ventricular; synchrony 
Submitted Jan 16, 2020. Accepted for publication Oct 23, 2020.

doi: $10.21037 /$ atm-20-839

View this article at: http://dx.doi.org/10.21037/atm-20-839

\section{Introduction}

Cardiac resynchronization therapy (CRT) is an important treatment modality following heart failure. CRT can coordinate contractions in all left ventricular (LV) segments, reducing valve reflux, increasing cardiac output per stroke, restoring cardiac electromechanical synchronization, reversing LV remodeling and improving heart failure symptoms. However, approximately $30 \%$ of patients receiving CRT have no response, and widespread CRT implementation is difficult due to its high technical requirements, cost, invasion, and radioactivity (1). Therefore, new methods are being explored to treat heart failure, improve LV synchronization and enhance heart systolic function. As a convenient, non-invasive, and radiation-free diagnosis and treatment modality, ultrasound has attracted increasing attention. Pre- and postoperative evaluation of synchrony and follow-up after CRT could be guided by echocardiography. However, as a new type of non-viral vector gene transfection, some progress has been made with research on ultrasound-targeted microbubble destruction (UTMD)-mediated exogenous gene transfection for the treatment of heart failure caused by myocardial infarction (MI), confirming that this method can promote angiogenesis, reduce myocardial fibrosis, and improve systolic function $(2,3)$. The mechanism may be that UTMD promotes an increase in cell membrane permeability, which allows the exogenous genes or drugs to enter the cell (4-6).

Furthermore, studies have also confirmed that twodimensional speckle-tracking echocardiography (2DSTE) technology can be used to evaluate the synchrony of cardiac motion. The LV wall can be divided into 6 parts: the anterior wall, anterior interventricular septum, posterior interventricular septum, lateral wall, inferior wall, and posterior wall. Each $\mathrm{LV}$ wall can be further divided into 3 segments: the basal segment, middle segment and apical segment. 2DSTE can be used to analyze the strain and strain rate of different segments for local systolic function. The standard deviation and the maximum difference of all segments' longitudinal strain were used as indicators to assess global systolic synchronicity. The previous studies were conducted from the perspective of structural reconstruction (7-9). In addition to structural reconstruction, sympathetic nerve reconstruction also showed important changes following heart failure. There are only a few published studies on cardiac sympathetic nerve reconstruction following UTMD treatment; therefore, the present study aimed to determine whether UTMD-mediated angiopoietin 1 (Ang1) gene transfection can improve angiogenesis and potentially reverse $\mathrm{LV}$ structural and sympathetic nerve remodeling in canines with MI.

UTMD-mediated Ang1 transfection was used to adjust neovascularization and neural fiber density around the infarcted myocardium in dogs. First, the UTMD-mediated transfection method was designed and performed to improve LV structural and sympathetic nerve remodeling, and then 2DSTE was used to evaluate the beneficial effects on myocardial synchrony and analyze the possible mechanisms. Our study provides a new non-invasive evaluation and treatment modality option for selected CRT non-responsive or refractory heart failure patients. We present the following article in accordance with the ARRIVE reporting checklist (available at http://dx.doi. org/10.21037/atm-20-839).

\section{Methods}

\section{Animals and the MI model}

Thirty adult male dogs $(15-21 \mathrm{~kg})$ were provided by the Animal Centre of Wuhan University (Wuhan, China). Experiments were performed under a project license (NO.: WDRM20150706) granted by the Ethical Committee of Renmin Hospital of Wuhan University, in compliance with the Guide for the Care and Use of Laboratory Animals.

All dogs were anesthetized before being intubated via the intramuscular administration of ketamine $(100 \mathrm{mg} / \mathrm{kg})$, followed by an intravenous injection of pentobarbital sodium $(30 \mathrm{mg} / \mathrm{kg}$; additional doses of $4 \mathrm{mg} / \mathrm{kg}$ were administered when necessary). The dogs were ventilated with ambient air supplemented with oxygen using a respirator (MAO01746; Harvard Apparatus, Holliston, MA, USA), and monitored with 12-lead electrocardiography (ECG). Cardiac catheterization was performed using a Philips FD20 Cath Lab Fluoroscopy Unit (Philips, Andover, 

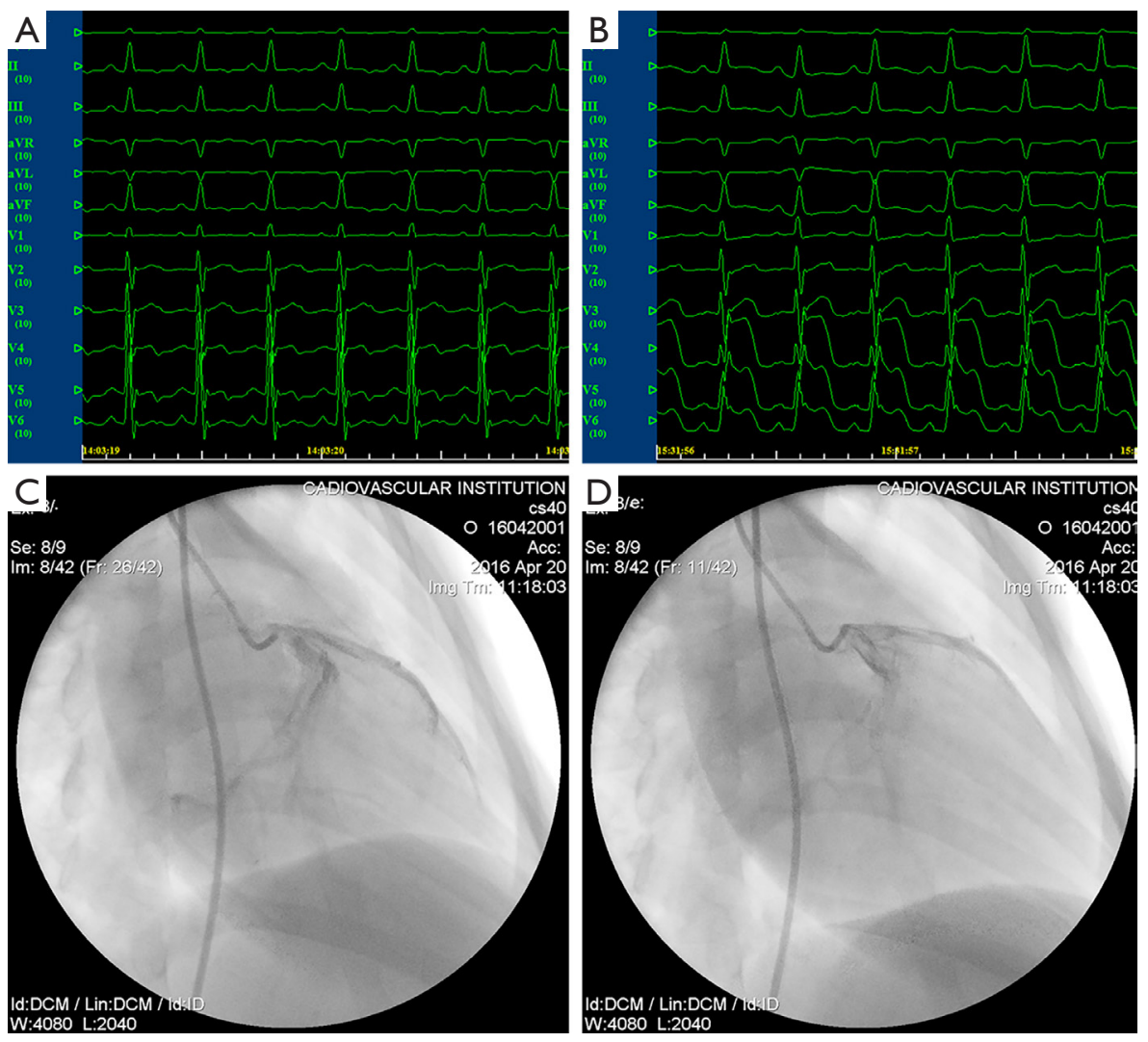

Figure 1 Process of establishing myocardial infarction model. (A) electrocardiogram before myocardial infarction (MI) modeling. (B) electrocardiogram after MI modeling; ST segment is elevated. (C) Coronary angiogram of left anterior descending coronary artery before establishing the MI model. (D) Coronary angiogram of left anterior descending coronary artery after establishing the MI model. Second coronary angiography confirmed ischemia in the embolism area.

MA, USA). A 6.0-Fr sheath was inserted from the right femoral artery. The dogs were systemically anticoagulated with 1,000 IU heparin. A 6.0-Fr angiographic catheter (TERUMO Radifocus OPTITORQUE, Tokyo, Japan) was introduced to select coronary angiograms of the left coronary artery. A 0.014 -inch coronary guide wire (TERUMO Runthrough NS, Tokyo, Japan) was inserted beyond the second diagonal branch of the left anterior descending coronary artery. The guide wire was removed when the 1.8-Fr. coronary microguide catheter (TERUMO Finecross MG, Tokyo, Japan) accessed to the site. Left anterior descending coronary artery occlusion was then immediately achieved via an endoluminal injection of 150-350 $\mu \mathrm{m}$ gelatin sponge particles (Alicon Pharm Sci \& Tec, Hangzhou, China). Finally, metoprolol ( $5 \mathrm{mg} / \mathrm{kg}$ ) and lignocaine $(3 \mathrm{mg} / \mathrm{kg})$ boluses were administered. The MI model was considered successful if significant ST-segment elevation was observed on ECG, and if the second coronary angiography confirmed ischemia in the embolism area (Figure 1).

\section{Plasmid construction}

The negative control plasmid vector sequence was CMVMCS-SV40-neomycin, with $\mathrm{X} b 0 \mathrm{I} / K p n \mathrm{I}$ as the cloning sites. The cloning of Ang1 primers were as follows: 5'- TACCGG ACTCAGATCTCGAGCGCCACCATGACAGTTTTC CTTTCCTTTG-3' (forward) and 5'-GATCCCGGGCC CGCGGTACCGTAAAATCTAAAGGTCGAATC ATC3' (reverse). The plasmids were amplified in Escherichia coli 
and isolated with the Wizard Maxiprep DNA Purification System (Promega, Madison, WI, USA). The concentration of the Ang1 plasmid was $0.5 \mathrm{mg} / \mathrm{mL}$.

\section{Preparation of microbubbles}

The preparation of microbubbles followed a previously described method (10). SonoVue contrast agent (Bracco, Geneva, Switzerland) lyophilized powder was reconstituted with $5 \mathrm{~mL} 0.9 \%$ sodium chloride solution. A suspension containing approximately $2.0 \times 10^{8}$ microbubbles $/ \mathrm{mL}$ was obtained after vigorous oscillation. The microbubbles were encapsulated by a phospholipid monolayer and filled with sulfur hexafluoride gas (diameter: $2.0-5.0 \mu \mathrm{m}$ ). The plasmid was then added to the microbubble suspension at 1: 1 and mixed for $2 \mathrm{~h}$ at $4^{\circ} \mathrm{C}$. The resulting upper layer consisted of microbubbles with attached plasmids; the bottom layer, which contained unattached plasmids, was discarded. The concentration of microbubbles with attached plasmids was approximately $1.5 \times 10^{8} / \mathrm{mL}$, the mean size was $2.4-7.0 \mu \mathrm{m}$, and the plasmid suspension concentration was $0.25 \mathrm{mg} / \mathrm{mL}$.

\section{UTMD-mediated Ang1 gene transfection}

Thirty dogs were randomly divided into three groups. Random sequence software was used to generate a set of random sequence numbers from 1 to 30 . Each dog was assigned a random sequence number, divided by 3 , and then grouped by the remainder. Dogs with the remainder of 1 entered the MI group ( $\mathrm{n}=10$, containing MI dogs without UTMD treatment), dogs with the remainder of 2 entered the UTMD group ( $\mathrm{n}=10$, containing MI dogs subjected to UTMD-mediated negative control plasmid treatment), and dogs with the remainder of 0 entered the UTMD-Ang1 group ( $\mathrm{n}=10$, containing MI dogs subjected to UTMD-mediated Ang1 plasmid treatment). A total of $2 \mathrm{~mL}$ microbubble suspension containing plasmids was injected via the elbow vein during ultrasound irradiation at an injection rate of $0.2 \mathrm{~mL} / \mathrm{min}$. The treatments were performed $24 \mathrm{~h}$ after the MI model was established. Insonation was performed in the heart region using an ultrasound gene transfection instrument with a $1-\mathrm{cm}-$ diameter transducer (Institute of Ultrasound Imaging of Chongqing Medical University, Chongqing, China). The ultrasound exposure involved intermittent continuous-wave irradiation for $10 \mathrm{~s}$ with 10 -s intervals for a total of $20 \mathrm{~min}$ at $300 \mathrm{kHz}$ and $2 \mathrm{~W} / \mathrm{cm}^{2}$.

\section{Echocardiography and LV systolic synchrony analysis by 2DSTE}

Transthoracic echocardiography was performed with a GE Vivid Q portable ultrasound machine (Vista, CA, USA) and an M4S-RS probe (frequency 2.0-3.5 MHz). Dynamic images of the parietal LV long axis and apical 4-chamber, 3-chamber, and 2-chamber sections were collected routinely in 5 consecutive cardiac cycles. The LV end-diastolic diameter (LVEDD) and LV end-systolic diameter (LVESD), interventricular septum thickness (IVS), LV posterior wall thickness (LVPW), and fractional shortening (FS) were measured in M-mode; the LV ejection fraction (LVEF) was calculated using Simpson's biplane method.

2DSTE technology with an EchoPac clinical workstation was used for the LV systolic synchrony analysis. The boundary between the inner and outer myocardial membrane was drawn and identified according to the software. Changes in the longitudinal strain $\left(S_{\text {mid }}\right.$ and $\left.\mathrm{S}_{\text {base }}\right)$ and strain rate $\left(\mathrm{SR}_{\text {mid }}\right.$ and $\left.\mathrm{SR}_{\text {base }}\right)$ of the $\mathrm{LV}$ segment (middle segment of the anterior and anteroseptal wall) and the adjacent segment (basal segment of the anterior and anteroseptal wall) were observed to evaluate the improvement in LV local systolic function. The standard deviation of all segments' longitudinal strain $\left(\mathrm{LS}_{\mathrm{sd}}\right)$ and the maximum difference of the longitudinal strain of all segments $\left(\mathrm{LS}_{\mathrm{dif}}\right)$ were used as indicators to assess the global systolic synchronicity.

\section{Immunobistochemistry for quantifying the microvessels and sympathetic nerve fibers}

On day 30 following MI, paraffin-embedded cryosections of the infarcted myocardium were used to determine the expression levels of CD31 (BS0468R; Beijing Biosynthesis Biotechnology, Beijing, China) and $\alpha$-smooth muscle actin ( $\alpha$-SMA; GB13036; Wuhan Goodbio Technology, Wuhan, China) and to evaluate the quantities of capillaries and arterioles by fluorescence microscopy. Tyrosine hydroxylase (TH) (AB93291; Abcam, Cambridge, UK) and growthassociated protein 43 (GAP43) (BS-0154R; BIOSS, MA, USA) were used to determine sympathetic nerve distribution and density. The 5 visual fields with the highest targeted tissue distribution were used to obtain the mean 
expression levels. Image-Pro Plus software was used to quantify the microvessels and nerve fibers.

\section{Western blot analysis}

The infarcted myocardial tissues were collected, and the Ang1, sarcoplasmic reticulum $\mathrm{Ca}^{2+}$-ATPase 2a (SERCA2a), and phospholamban (PLB) proteins were extracted and then separated via $10 \%$ sodium dodecyl sulfatepolyacrylamide gel electrophoresis according to previously described standard procedures (11). The separated proteins were transferred to polyvinylidene fluoride membranes and incubated with primary antibodies (Abcam Inc., Cambridge, MA, USA) at a 1:1,000 dilution overnight at $4{ }^{\circ} \mathrm{C}$. The membrane was then blocked with $10 \%$ skimmed milk at room temperature for $1 \mathrm{~h}$, and the appropriate secondary antibodies were used at a 1:3,000 dilution for $1 \mathrm{~h}$ at room temperature. The proteins were detected via chemiluminescence, quantified by densitometry using Scion Image software (Scion, Frederick, MD, USA), and then normalized glyceraldehyde-3-phosphate dehydrogenase (GAPDH) as a loading control.

\section{Blood sampling}

Venous blood samples were collected in ice-chilled tubes coated with ethylenediaminetetraacetic acid (EDTA; BD Vacutainer K2E; BD Diagnostics, NJ, USA) on the day before $\mathrm{MI}$ and day 1, 7, and 30 following MI. The plasma was separated by centrifugation at 3,000 rpm for $10 \mathrm{~min}$ at $4{ }^{\circ} \mathrm{C}$ and stored at $-80{ }^{\circ} \mathrm{C}$ until assayed. Norepinephrine (NE) and $\mathrm{N}$-terminal pro-B-type-natriuretic peptide (NT-proBNP) concentrations were determined with a radioimmunoassay kit from the American Laboratory Products Company (Salem, NH, USA) according to the manufacturer's procedure.

\section{Statistical analysis}

SPSS version 19.0 (SPSS, Armonk, NY, USA) was used. All measurement data are expressed as mean \pm standard deviation. ImageJ software was used to quantify the results of histochemical staining. Normality tests and homogeneity of variance tests were performed for all data. One-way analysis of variance was used if the data exhibited a normal distribution and homogeneity of variance. After a statistically significant difference was identified, the least significant difference (LSD) method was used for intergroup comparisons. The Kruskal-Wallis H test (rank sum test) was used in cases of non-normal distribution or unequal variance. $\mathrm{P}<0.05$ indicated statistical significance.

\section{Results}

\section{Survival rates of the three groups of dogs}

In the MI group, 3 dogs died and 7 dogs survived after the MI model was established. In the UTMD group and the UTMD-Ang1 group, 2 dogs died, and 8 dogs survived. The survival rates showed no significant difference among the groups.

\section{Analysis of conventional and echocardiographic parameters}

There were no significant differences in body mass, heart rate, or LVPW among the three groups $(\mathrm{P}>0.05)$. Compared with the MI group, the UTMD-Ang1 group exhibited increased LVEDD and LVESD values and decreased IVS, LVEF, and FS values (all $\mathrm{P}<0.05$, Table 1).

\section{Analysis of myocardial synchrony parameters}

Local systolic function indexes, $\mathrm{S}_{\text {mid }}$ and $\mathrm{SR}_{\text {mid, }}$ and overall synchrony parameters, $\mathrm{LS}_{\mathrm{sd}}$ and $\mathrm{LS}_{\mathrm{dif}}$, were significantly different among the three groups $(\mathrm{P}<0.05)$. Contraction movement in the $\mathrm{MI}$ group was more asynchronous, while local contraction was enhanced and global synchronization was improved in the UTMD and UTMD-Ang1 groups $(\mathrm{P}<0.05)$. There were no significant differences in $\mathrm{S}_{\text {base }}$ or $\mathrm{SR}_{\text {base }}$ among the three groups ( $\left.\mathrm{P}>0.05\right)$ (Table 2 and Figure 2$)$.

\section{Analysis of the density of neovessels around the MI area}

CD31 and $\alpha$-SMA immunohistochemical staining showed that the density of capillaries in the MI group was lower than that in the UTMD group, while the density in the UTMD-Ang1 group was significantly higher than that in the UTMD group $(\mathrm{P}<0.05)$ (Figure 3).

\section{Analysis of the density of peripheral nerve fibers around the MI area}

Compared with the MI group, the UTMD-Ang1 group exhibited a significantly lower density of TH- and GAP43costained sympathetic nerve fibers in the area surrounding 
Table 1 Comparison of left ventricular structural and systolic function in the three groups

\begin{tabular}{llll}
\hline Parameters & Ml group $(\mathrm{n}=7)$ & UTMD group $(\mathrm{n}=8)$ & UTMD-Ang1 group $(\mathrm{n}=8)$ \\
\hline Weight $(\mathrm{kg})$ & $16.8 \pm 2.5$ & $17.5 \pm 2.1$ & $17.1 \pm 2.3$ \\
HR $(\mathrm{bpm})$ & $135 \pm 15$ & $132 \pm 18$ & $134 \pm 16$ \\
LVEDD $(\mathrm{mm})$ & $34.5 \pm 2.2$ & $34.1 \pm 2.8$ & $31.2 \pm 2.3^{\mathrm{ab}}$ \\
LVESD $(\mathrm{mm})$ & $28.0 \pm 1.7$ & $27.0 \pm 2.3$ & $25.2 \pm 1.5^{\mathrm{ab}}$ \\
IVS $(\mathrm{mm})$ & $3.1 \pm 0.2$ & $3.3 \pm 0.3$ & $3.3 \pm 0.3$ \\
LVPW (mm) & $6.9 \pm 0.2$ & $6.8 \pm 0.3$ & $7.0 \pm 0.3$ \\
LVEF $(\%)$ & $37.3 \pm 3.1$ & $39.3 \pm 4.5$ & $44.6 \pm 4.3^{\mathrm{ab}}$ \\
FS $(\%)$ & $18.7 \pm 2.1$ & $19.7 \pm 3.2$ & $21.8 \pm 2.0^{\mathrm{ab}}$ \\
\hline
\end{tabular}

${ }^{\mathrm{a} C}$ Compared with the MI group $(\mathrm{P}<0.05)$. ${ }^{\mathrm{b} C o m p a r e d}$ with the UTMD group $(\mathrm{P}<0.05)$. LV, left ventricular; MI, myocardial infarction; UTMD, ultrasound-targeted microbubble destruction; HR, heart rate; LVEDD, left ventricular end-diastolic diameter; LVESD, left ventricular endsystolic diameter; IVS, interventricular septum thickness; LVPW, left ventricular posterior wall thickness; LVEF, left ventricular ejection fraction; and FS, fractional shortening.

Table 2 Comparison of left ventricular synchrony parameters in the three groups

\begin{tabular}{llcl}
\hline Parameters & Ml group $(n=7)$ & UTMD group $(n=8)$ & UTMD-Ang1 group $(n=8)$ \\
\hline $\mathrm{S}_{\text {mid }}(\%)$ & $-7.3 \pm 2.2$ & $-8.7 \pm 2.5$ & $-10.5 \pm 2.4^{\mathrm{a}}$ \\
$\mathrm{SR}_{\text {mid }}\left(\mathrm{S}^{-1}\right)$ & $-16.9 \pm 4.7$ & $-19.8 \pm 5.4$ & $-23.8 \pm 5.9^{\mathrm{a}}$ \\
$\mathrm{S}_{\text {base }}(\%)$ & $-20.1 \pm 5.1$ & $-19.2 \pm 4.8$ & $-18.2 \pm 5.0$ \\
$\mathrm{SR}_{\text {base }}\left(\mathrm{s}^{-1}\right)$ & $-45.3 \pm 7.1$ & $-43.7 \pm 7.5$ & $-41.8 \pm 6.9$ \\
$\mathrm{LS}_{\text {sd }}(\mathrm{ms})$ & $65.3 \pm 16.3$ & $41.8 \pm 10.3^{\mathrm{a}}$ & $30.5 \pm 10.7^{\mathrm{ab}}$ \\
$\mathrm{LS}_{\text {dif }}(\%)$ & $26.7 \pm 8.9$ & $18.2 \pm 4.5^{\mathrm{a}}$ & $13.5 \pm 4.1^{\mathrm{ab}}$ \\
\hline
\end{tabular}

${ }^{\mathrm{a} C}$ Compared with the MI group $(\mathrm{P}<0.05)$. ${ }^{\mathrm{b} C o m p a r e d}$ with the UTMD group $(\mathrm{P}<0.05)$. LV, left ventricular; MI, myocardial infarction; UTMD, ultrasound-targeted microbubble destruction; $\mathrm{S}_{\text {mid }}$, the strain of all the middle segments of LV; $\mathrm{SR}_{\text {mid }}$, the strain rate of all the middle segments of LV; $\mathrm{S}_{\text {base }}$, the strain of all the base segments of LV; $\mathrm{SR}_{\text {base }}$, the strain rate of all the base segments of LV; LS ${ }_{\text {sd }}$, the standard deviation of all segments' longitudinal strain; and LS $_{\text {dif }}$, the maximum difference of all segments' longitudinal strain.

the infarcted myocardium $(\mathrm{P}<0.05)$ (Figure 4).

\section{Analysis of Ang1 and key $\mathrm{Ca}^{2+}$ homeostasis-related proteins by Western blot}

Western blot showed that Ang1 and SERCA2a protein levels were significantly higher in the UTMD-Ang1 group than the MI and UTMD groups $(\mathrm{P}<0.05)$. In contrast, PLB levels were higher in the MI group and lowered in the UTMD-Ang1 group compared with the UTMD group $(\mathrm{P}<0.05)$ (Figure 5).

\section{Analysis of plasma NE and NT-proBNP levels}

Plasma NE and NT-proBNP levels were increased from day 1 to 30 following MI. However, the UTMD-Ang1 group exhibited significantly reduced plasma NE and NTproBNP levels compared with the MI and UTMD groups $(\mathrm{P}<0.05)$ (Figure 6).

\section{Discussion}

During heart failure, all LV segments and walls between the left and right ventricles are often not synchrony, 

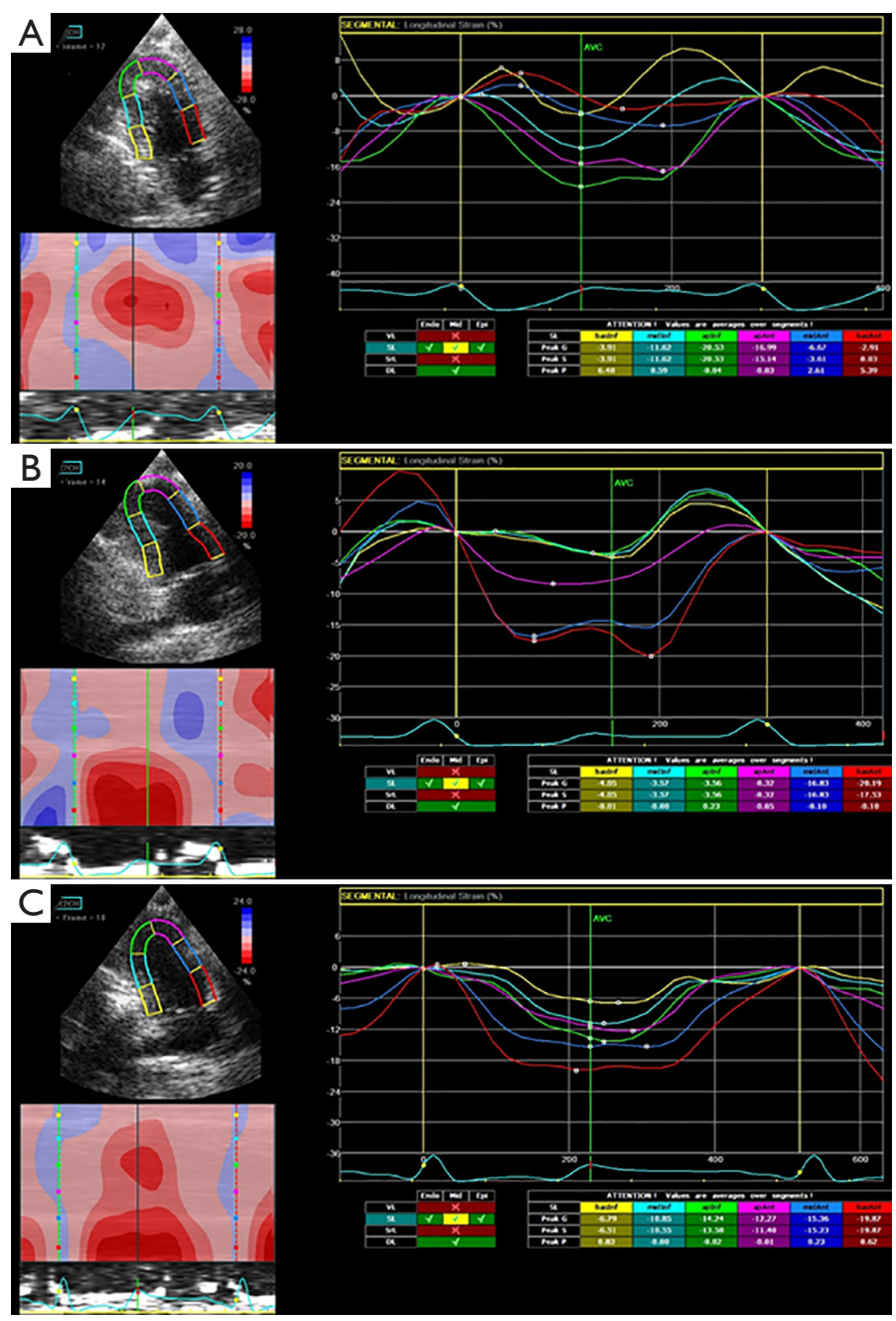

Figure 2 Two-dimensional speckle tracking echocardiography showed the left ventricular longitudinal strain curve of the apical 2-chamber section view. (A) All segments were disordered in motion in the myocardial infarction (MI) group, and the peak time was significantly different, suggesting that the myocardial motion is not in synchronization. (B) Coordination of each segment's peak time was slightly improved in the ultrasound-targeted microbubble destruction (UTMD) group. (C) Synchronization of each segment was significantly improved and tended to be consistent following UTMD-Ang1 treatment.

leading to cardiac hemodynamic disorders. In particular, a lack of coordination in LV segments' movement results in ineffective contractions, decreased LV stroke volume, and increased residual blood volume at the end of systole, aggravating heart failure. In 2014, the European Society of Cardiology guidelines for the diagnosis and treatment of heart failure placed strict restrictions on CRT indications for patients, but the high non-response rate for CRT persisted (12). However, new methods are being explored to treat heart failure, improve LV synchronization, and enhance heart systolic function. Some progress has been made in research on UTMD-mediated exogenous gene transfection to treat refractory heart failure by promoting the increase in cell membrane permeability, allowing the exogenous genes to enter the cell for transfection $(4,6)$. For example, Fujii et al. found that non-invasive UTMD successfully targets stem cell growth factor and stromal cellderived factor genes in the infarcted myocardia of mice, increases the density of blood vessels, improves myocardial perfusion, and enhances LV systolic function (13). This is 
A

CD31

$\alpha-S M A$

DAPI
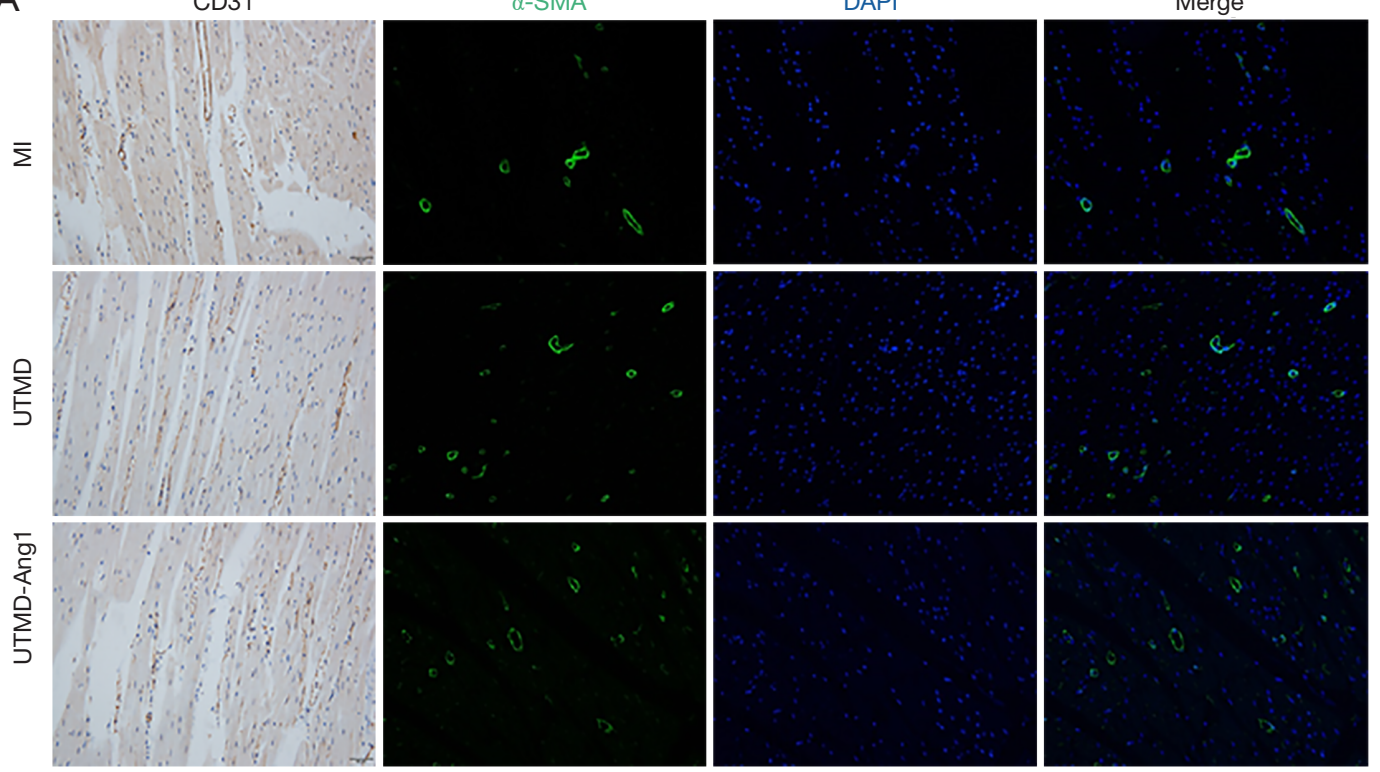

B
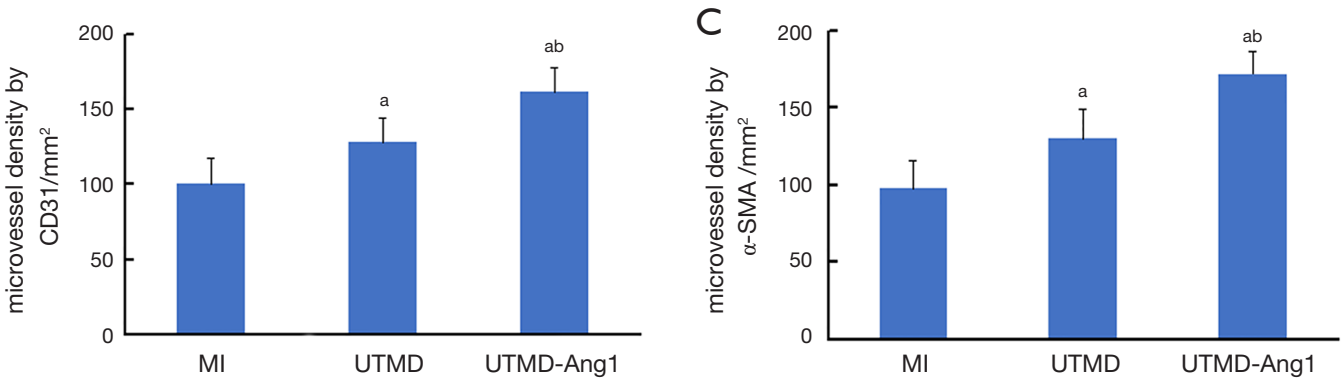

Figure 3 Analysis of the density of neovessels around the myocardial infarction (MI) area. (A) Brown indicates the vascular endothelium with CD31, and green indicates the vascular endothelium with $\alpha$-smooth muscle actin $(\alpha-\mathrm{SMA})(\times 200$, scale bar $=50 \mu \mathrm{m})$. Nucleus showing 4',6'-diamidino-2-phenylindole dihydrochloride (DAPI) staining (blue). Merge was composed by $\alpha$-SMA and DAPI. (B,C) Comparison of the blood vessels density in the three groups based on CD31 and $\alpha$-SMA. ${ }^{a}$ Compared with the MI group $(\mathrm{P}<0.05)$. ${ }^{b}$ Compared with the ultrasound-targeted microbubble destruction (UTMD) group $(\mathrm{P}<0.05)$.

mainly due to the cavitation effect of UTMD, which could promote the increase in cell membrane permeability and allow exogenous genes or drugs to enter the cell (4-6). Therefore, non-invasive UTMD is an important method to promote angiogenesis after MI.

Ang1, a growth factor peptide that plays an important role in angiogenesis, can effectively promote the proliferation, differentiation and migration of vascular endothelial cells and mediate intercellular adhesion to form new microvascular lumens (14). Rufaihah et al. used a combination of vascular endothelial growth factor and Ang1 to treat heart failure caused by acute $M I$ in rats and found that combining these factors could significantly improve angiogenesis and LV systolic function (15). We found that UTMD could successfully mediate Ang1 gene transfection for rabbits with $M I$ in our previous study (16). In the present study, UTMD-mediated Ang1 gene transfection was further proven to promote angiogenesis and improve systolic function effectively.

It has been proven that 2DSTE can be used to evaluate the synchronization of the heart (7-9). 2DSTE can also distinguish the degree of strain in the endocardium or epicardium after myocardial ischemia, and can also be used to evaluate synchrony in dogs $(17,18)$. In the present study, there were statistically significant differences in the local and total strain and synchrony parameters before and after 
A
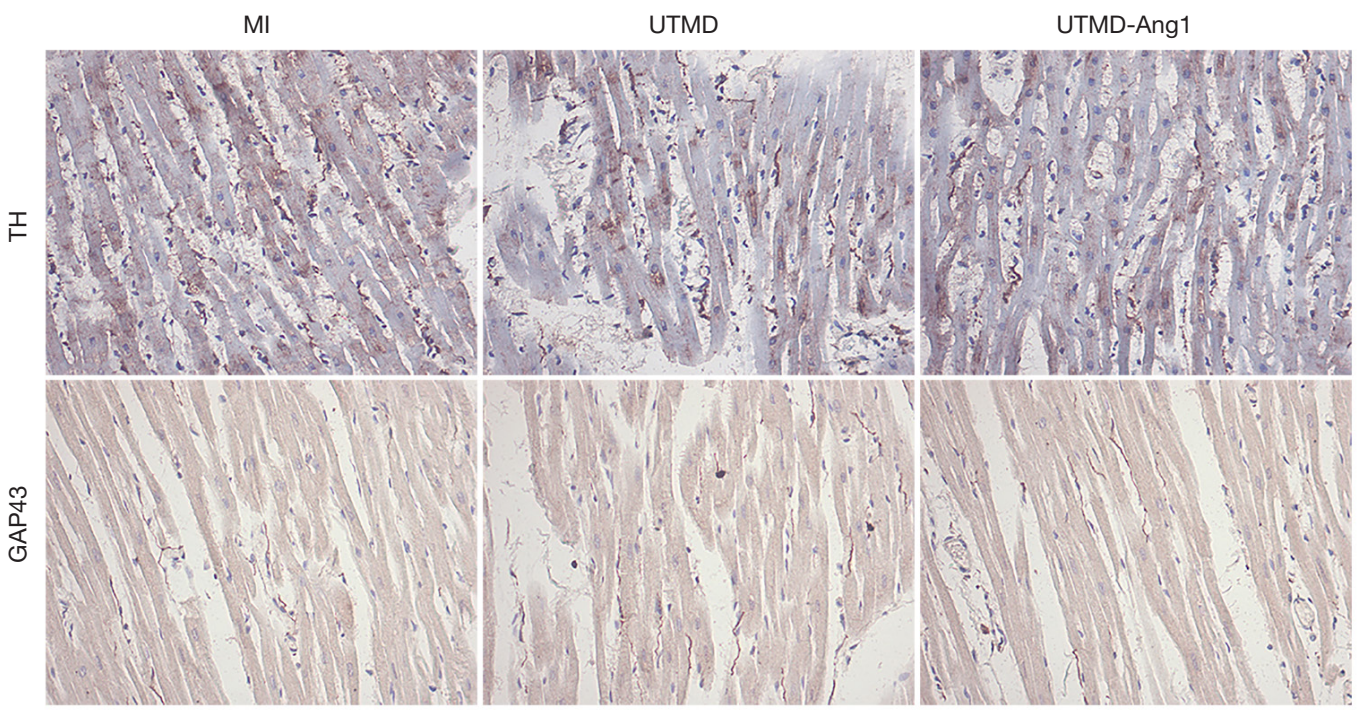

B
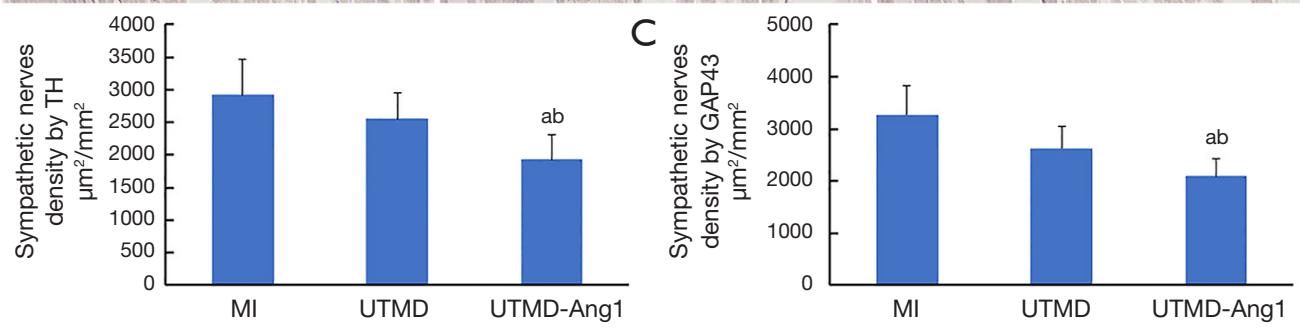

Figure 4 Analysis of the density of sympathetic nerve fibers around the myocardial infarction (MI) area. (A) Brown indicates the nerver fibers with tyrosine hydroxylase (TH) or growth-associated protein 43 (GAP43) $(\times 200$, scale bar $=50 \mu \mathrm{m})$. (B, C) Comparison of the nerver fibers density in the three groups based on TH and GAP43. ${ }^{\mathrm{a} C o m p a r e d ~ w i t h ~ t h e ~} \mathrm{MI}$ group $(\mathrm{P}<0.05)$. ${ }^{\mathrm{b}} \mathrm{Compared}$ with the ultrasound-targeted microbubble destruction (UTMD) group $(\mathrm{P}<0.05)$.

MI treatment, possibly because myocyte ischemia, hypoxia, edema, and necrosis after MI and metabolic dysfunction impaired local myocardial systolic function and extended the time to peak strain in the corresponding myocardial segment, therefore causing asynchronous contractions. After UTMD-mediated Ang1 transfection, angiogenesis was induced, and blood supply to the myocardium might have been partially restored. The contractility of the myocardial segment was correspondingly enhanced, and LV synchronization was improved.

Myocardial contractility is closely related to the roles of SERCA2a and PLB, which are key $\mathrm{Ca}^{2+}$ movement proteins that regulate myocardial excitation-contraction coupling. SERCA2a plays a critical role in regulating cytoplasmic $\mathrm{Ca}^{2+}$ concentrations. When PLB is dephosphorylated, it binds to SERCA2a to form a complex, reducing the affinity of SERCA2a for $\mathrm{Ca}^{2+}$ ions. When PLB is phosphorylated, the complex dissociates, and its affinity for $\mathrm{Ca}^{2+}$ ions increases
(19). Ischemia and hypoxia in myocardial cells decrease the expression of the SERCA2a protein and the phosphorylation of PLB in heart failure patients, impairing $\mathrm{Ca}^{2+}$ pump function and leading to myocardial contraction dysfunction. When large numbers of angiogenic events occur, the oxygen supply to myocardial cells improves, SERCA2a protein expression and PLB phosphorylation levels increase, pump function recovers, the systolic ability is enhanced and synchronicity is improved. Similarly, Cordero-Reyes et al., who studied molecular and cellular correlates of LV systolic and diastolic function with 2DSTE, revealed that SERCA2a plays an important role in improving the systolic function of myocardial cells (20). Other studies have suggested that therapeutic ultrasound could enhance the opening of $\mathrm{Ca}^{2+}$ channels on the endothelial cell membrane and increase intracellular $\mathrm{Ca}^{2+}$ concentrations $(21,22)$. In the present study, the combined effects of the increases in key proteins related to $\mathrm{Ca}^{2+}$ homeostasis and the concentrations of $\mathrm{Ca}^{2+}$ 
A

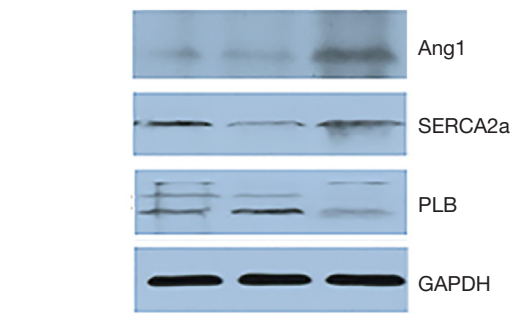

C

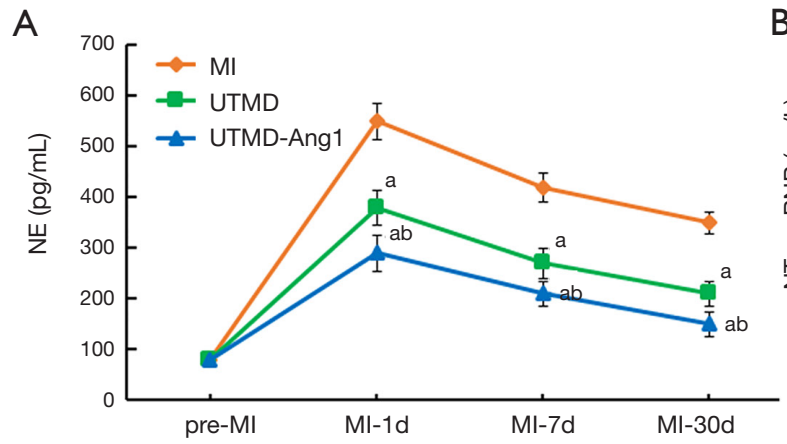
ultrasound-targeted microbubble destruction (UTMD) group $(\mathrm{P}<0.05)$.
B

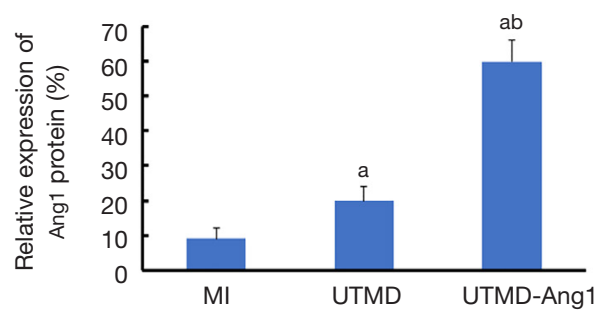

D

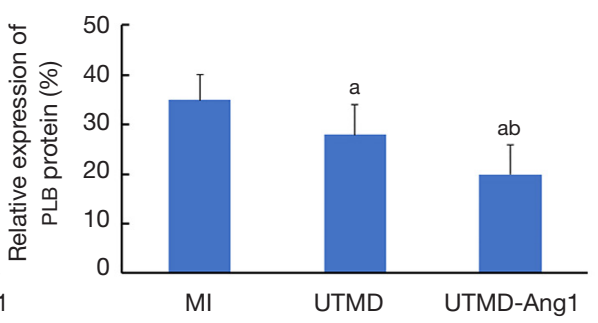

Figure 5 Analysis of proteins by Western blot. (A) protein bands of Ang1, sarcoplasmic reticulum $\mathrm{Ca}^{2+}$-ATPase 2a (SERCA2a), phospholamban (PLB) and glyceraldehyde-3-phosphate dehydrogenase (GAPDH) revealed by Western blot. (B-D) Comparison of proteins in the three groups. GAPDH normalized the results. ${ }^{\mathrm{a} C o m p a r e d ~ w i t h ~ t h e ~ m y o c a r d i a l ~ i n f a r c t i o n ~(M I) ~ g r o u p ~}(\mathrm{P}<0.05) .{ }^{\mathrm{b}} \mathrm{Compared}$ with the

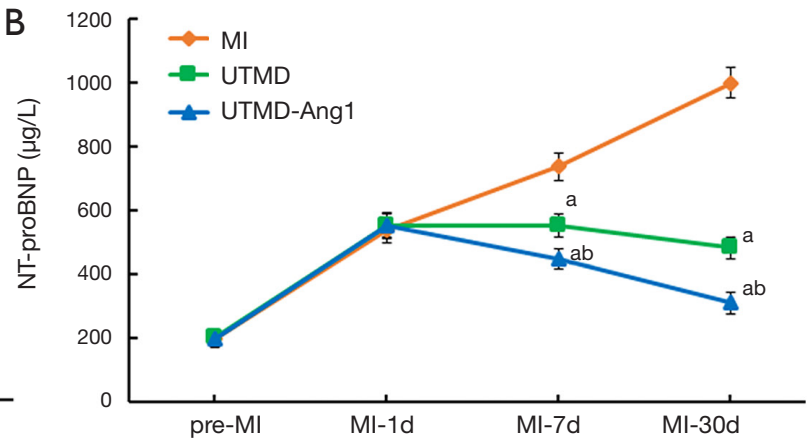

Figure 6 Analysis of plasma norepinephrine (NE) and N-terminal pro-B-type-natriuretic peptide (NT-proBNP) levels. NE and NT-proBNP levels were detected at different times before and after myocardial infarction (MI). ${ }^{a}$ Compared with the MI group $(\mathrm{P}<0.05) .{ }^{b}$ Compared with the ultrasound-targeted microbubble destruction (UTMD) group $(\mathrm{P}<0.05)$.

led to enhanced contractility of myocardial cells in the peripheral area of infarction, significantly improving LV synchronization and the LV global systolic function.

Structural remodeling is the basis of chronic heart failure after MI. To assess the degree of sympathetic nerve remodeling, sympathetic nerve growth activity and distribution after MI can be evaluated through immunohistochemical determination of the density of $\mathrm{TH}$ and GAP43 staining in myocardial tissue (23). TH is a rate- limiting enzyme synthesized by sympathetic cytoplasmic catecholamines that can be used as a marker to detect the distribution of mature sympathetic nerves. GAP43 is a specific protein synthesized by the cell bodies of neurons in the axons, where neurons grow outwardly during development. GAP43-positive staining density reflects the growth of nerve fibers, and GAP43 expression is low in mature neurons; therefore, it can be used as a marker of nerve damage and repair. The combination of GAP43 
and TH can reflect neural development and regeneration. Local autonomic nerve damage occurs in the context of MI. A reaction involving nerve repair of nervous sheath cells and excessive axon regeneration occurs, especially surrounding the infarcted area. Increased sympathetic nerve activity following MI can promote LV remodeling, while sympathetic activity inhibition can improve cardiac function and reverse remodeling (24).

In our study, TH and GAP43 expression was upregulated in the peripheral area in the MI group, suggesting obvious nerve fiber regeneration. In contrast, the expression of TH and GAP43 in the UTMD-Ang1 group decreased, likely for two reasons. First, UTMD-Ang1 targeted therapy can promote angiogenesis, reduce excessive nerve fiber regeneration, and reverse nerve remodeling after MI by improving the microenvironment, inhibiting the expression of inflammatory factors, and enhancing the self-repair ability of nerve cells $(3,25)$. Second, ultrasonic irradiation can directly stimulate nerve fiber cells and directly regulate central nervous system and peripheral neuron function through thermal and mechanical effects (26); therefore, it can regulate nerve fiber activity to change the physiological functions of specific regions. Also, increases in plasma NE levels and cardiac sympathetic nerve activity can induce myocardial remodeling (27), and the extent to which plasma NE concentrations are elevated correlates directly with the severity of LV dysfunction $(28,29)$. Evidence suggests that patients with high levels of NT-proBNP are at higher risk of LV remodeling (30). In our study, UTMDAng1 decreased plasma NE and NT-proBNP levels. In other words, UTMD-Ang1 can be used to adjust nerve remodeling concerning the number and activity of nerve fibers.

\section{Limitations}

First, due to laboratory limitations, isolated perfusion of large animals' hearts was not implemented, and $\mathrm{Ca}^{2+}$ in the sarcoplasmic reticulum of cardiomyocytes was not directly detected; the analysis of $\mathrm{Ca}^{2+}$ concentrations could have more directly reflected the excitation-contraction coupling activity of cardiomyocytes. Second, therapeutic ultrasound can increase intracellular $\mathrm{Ca}^{2+}$ concentrations, but the underlying mechanism by which ultrasound irradiation changes $\mathrm{Ca}^{2+}$ concentrations in the sarcoplasmic reticulum of cardiomyocytes is still not clear, and further studies are needed. Aside from structural and nervous remodeling, the effect of electromechanical coupling of myocardial contractions on synchronization has not been studied. However, electromechanical coupling plays an important role in the excitation-contraction coupling of cardiomyocytes.

\section{Conclusions}

UTMD-mediated Ang1 gene transfection can promote peripheral angiogenesis in the infarcted myocardium, reverse $L V$ structural and nervous remodeling, and improve synchronization. These effects may be related to the expression of the key $\mathrm{Ca}^{2+}$ homeostasis-related proteins SERCA2a and PLB, and the nerve fiber proteins, $\mathrm{TH}$ and GAP43.

\section{Acknowledgments}

Funding: This study was supported by the National Natural Science Foundation of China (81901757, 81901759 and 81971624) and the Nature Science Foundation of Hubei Province (No. ZRM S2019000334).

\section{Footnote}

Reporting Checklist: The authors have completed the ARRIVE reporting checklist. Available at http://dx.doi. org/10.21037/atm-20-839

Data Sharing Statement: Available at http://dx.doi. org/10.21037/atm-20-839

Conflicts of Interest: All authors have completed the ICMJE uniform disclosure form (available at http://dx.doi. org/10.21037/atm-20-839). The authors have no conflicts of interest to declare.

Ethical Statement: The authors are accountable for all aspects of the work in ensuring that questions related to the accuracy or integrity of any part of the work are appropriately investigated and resolved. All animals were provided by the Animal Centre of Wuhan University. Experiments were performed under a project license (No. WDRM20150706) granted by the Ethical Committee of Renmin Hospital of Wuhan University, in compliance with the Guide for the Care and Use of Laboratory Animals.

Open Access Statement: This is an Open Access article distributed in accordance with the Creative Commons 
Attribution-NonCommercial-NoDerivs 4.0 International License (CC BY-NC-ND 4.0), which permits the noncommercial replication and distribution of the article with the strict proviso that no changes or edits are made and the original work is properly cited (including links to both the formal publication through the relevant DOI and the license). See: https://creativecommons.org/licenses/by-nc-nd/4.0/.

\section{References}

1. Brignole M, Auricchio A, Baron-Esquivias G, et al. 2013 ESC guidelines on cardiac pacing and cardiac resynchronization therapy: the task force on cardiac pacing and resynchronization therapy of the European Society of Cardiology (ESC). Developed in collaboration with the European Heart Rhythm Association (EHRA). Eur Heart J 2013;34:2281-329.

2. Liao YY, Chen ZY, Wang YX, et al. New progress in angiogenesis therapy of cardiovascular disease by ultrasound targeted microbubble destruction. Biomed Res Int 2014;2014:872984.

3. Ling ZY, Shu SY, Zhong SG, et al. Ultrasound targeted microbubble destruction promotes angiogenesis and heart function by inducing myocardial microenvironment change. Ultrasound Med Biol 2013;39:2001-10.

4. Song Y, Xie X, Gao Y, et al. Ultrasound-induced microbubble destruction promotes targeted delivery of adipose-derived stem cells to improve hind-limb ischemia of diabetic mice. Am J Transl Res 2016;8:2585-96.

5. Lamy R, Chan E, Lee OT, et al. $880 \mathrm{kHz}$ ultrasound treatment for drug delivery to the vitreous humor. Am J Transl Res 2018;10:3162-70.

6. Wang XL, Qi J, Shi YQ, et al. Atorvastatin plus therapeutic ultrasound improve postnatal neovascularization in response to hindlimb ischemia via the PI3K-Akt pathway. Am J Transl Res 2019;11:2877-86.

7. Heydari B, Jerosch-Herold M, Kwong RY. Imaging for planning of cardiac resynchronization therapy. JACC Cardiovasc Imaging 2012;5:93-110.

8. Hu B, Zhou Q, Chen J, et al. Prediction for Improvement and Remodeling in First-Onset Myocardial Infarction by Speckle Tracking Echocardiography: Is Global or Regional Selection Better? Ultrasound Med Biol 2017;43:2452-60.

9. Collier P, Phelan D, Klein A. A Test in Context: Myocardial Strain Measured by Speckle-Tracking Echocardiography. J Am Coll Cardiol 2017;69:1043-56.

10. Shohet RV, Chen S, Zhou YT, et al. Echocardiographic destruction of albumin microbubbles directs gene delivery to the myocardium. Circulation 2000;101:2554-6.

11. McClatchy DB, Ma Y, Liem DA, et al. Quantitative temporal analysis of protein dynamics in cardiac remodeling. J Mol Cell Cardiol 2018;121:163-72.

12. McMurray JJ, Adamopoulos S, Anker SD, et al. ESC Guidelines for the diagnosis and treatment of acute and chronic heart failure 2012: The Task Force for the Diagnosis and Treatment of Acute and Chronic Heart Failure 2012 of the European Society of Cardiology. Developed in collaboration with the Heart Failure Association (HFA) of the ESC. Eur Heart J 2012;33:1787-847.

13. Fujii $\mathrm{H}, \mathrm{Li} \mathrm{SH}, \mathrm{Wu}$ J, et al. Repeated and targeted transfer of angiogenic plasmids into the infarcted rat heart via ultrasound targeted microbubble destruction enhances cardiac repair. Eur Heart J 2011;32:2075-84.

14. Peirce SM, Price RJ, Skalak TC. Spatial and temporal control of angiogenesis and arterialization using focal applications of VEGF164 and Ang-1. Am J Physiol Heart Circ Physiol 2004;286:H918-25.

15. Rufaihah AJ, Johari NA, Vaibavi SR, et al. Dual delivery of VEGF and ANG-1 in ischemic hearts using an injectable hydrogel. Acta Biomater 2017;48:58-67.

16. Deng Q, Hu B, Cao S, et al. Improving the efficacy of therapeutic angiogenesis by UTMD-mediated Ang-1 gene delivery to the infarcted myocardium. Int J Mol Med 2015;36:335-44.

17. Corda A, Pinna Parpaglia ML, Sotgiu G, et al. Use of 2-dimensional speckle-tracking echocardiography to assess left ventricular systolic function in dogs with systemic inflammatory response syndrome. J Vet Intern Med 2019;33:423-31.

18. Hu W, Zhao QY, Yu SB, et al. Renal sympathetic denervation inhibits the development of left ventricular mechanical dyssynchrony during the progression of heart failure in dogs. Cardiovasc Ultrasound 2014;12:47.

19. Schmitt JP, Ahmad F, Lorenz K, et al. Alterations of phospholamban function can exhibit cardiotoxic effects independent of excessive sarcoplasmic reticulum $\mathrm{Ca} 2+-$ ATPase inhibition. Circulation 2009;119:436-44.

20. Cordero-Reyes AM, Youker K, Estep JD, et al. Molecular and cellular correlates of cardiac function in end-stage DCM: a study using speckle tracking echocardiography. JACC Cardiovasc Imaging 2014;7:441-52.

21. Hassan MA, Campbell P, Kondo T. The role of Ca2+ in ultrasound-elicited bioeffects: progress, perspectives and prospects. Drug Discov Today 2010;15:892-906.

22. Lu ZY, Li RL, Zhou HS, et al. Rescue of hypertension- 
related impairment of angiogenesis by therapeutic ultrasound. Am J Transl Res 2016;8:3087-96.

23. Yuan MJ, Huang CX, Tang YH, et al. A novel peptide ghrelin inhibits neural remodeling after myocardial infarction in rats. Eur J Pharmacol 2009;618:52-7.

24. Chen T, Cai MX, Li YY, et al. Aerobic exercise inhibits sympathetic nerve sprouting and restores $\beta$-adrenergic receptor balance in rats with myocardial infarction. PLoS One 2014;9:e97810.

25. Wang Z, Yu L, Huang B, et al. Low-level transcutaneous electrical stimulation of the auricular branch of vagus nerve ameliorates left ventricular remodeling and dysfunction by downregulation of matrix metalloproteinase 9 and transforming growth factor $\beta 1$. J Cardiovasc Pharmacol 2015;65:342-8.

26. Downs ME, Lee SA, Yang G, et al. Non-invasive peripheral nerve stimulation via focused ultrasound in vivo. Phys Med Biol 2018;63:035011.

Cite this article as: Cao S, Deng Q, Wang Y, Zhou Y, Zhou Q. Ultrasound-targeted microbubble destruction-mediated Ang1 gene transfection improves left ventricular structural and sympathetic nerve remodeling in canines with myocardial infarction. Ann Transl Med 2021;9(3):221. doi: 10.21037/atm$20-839$
27. Wang Z, Yu L, Wang S, et al. Chronic intermittent lowlevel transcutaneous electrical stimulation of auricular branch of vagus nerve improves left ventricular remodeling in conscious dogs with healed myocardial infarction. Circ Heart Fail 2014;7:1014-21.

28. Kaye DM, Lefkovits J, Jennings GL, et al. Adverse consequences of high sympathetic nervous activity in the failing human heart. J Am Coll Cardiol 1995;26:1257-63.

29. Swedberg K, Eneroth P, Kjekshus J, et al. Hormones regulating cardiovascular function in patients with severe congestive heart failure and their relation to mortality. CONSENSUS Trial Study Group. Circulation 1990;82:1730-6.

30. Fertin M, Dubois E, Belliard A, et al. Usefulness of circulating biomarkers for the prediction of left ventricular remodeling after myocardial infarction. Am J Cardiol 2012;110:277-83. 